\title{
The Status of Safety Management Systems at Collegiate Flight Training Institutions
}

\author{
Michael Robertson
}

Matt Romero

\author{
Steve Goetz
}

Southern Illinois University

\begin{abstract}
The purpose of this study was to determine the current status of SMS development and implementation at Collegiate Flight Training Organizations across the United States. Research questions address the following: What are the SMS demographics of collegiate flight schools? What level of organizational support is reported for SMS by collegiate flight schools? What progress is being made toward the development or implementation of the components of SMS at collegiate flight schools? The majority of the collegiate flight training provider's responses indicated some level of SMS implementation and overall indicate more engagement with SMS than other areas of the aviation industry.
\end{abstract}

\section{Introduction}

Safety Management Systems (SMS) is permeating the aviation industry. Airlines, air traffic, airports, flight training organizations, and other parts of the industry are all incorporating the SMS risk-based approach to safety management, and it is important to understand how the various parts of the aviation industry are incorporating elements of the new approach to safety management. The International Civil Aviation Organization (ICAO) defines SMS as "an organized approach to managing safety, including the necessary organizational structures, accountabilities, policies, and procedures" (ICAO, 2013, pp. 1-2). The Federal Aviation Administration (FAA) defines SMS as, "the formal, top-down, organization-wide approach to managing safety risk and assuring the effectiveness of safety risk controls. It includes systematic procedures, practices, and policies for the management of safety risk" (FAA, 2015a). An SMS requires the organization to examine its operations and the decisions made regarding those operations, which allows an organization to adapt to changes and promotes continuous improvement of safety at the organizational level (FAA, 2015a).

According to the Pilot Career Center (2016), there are over 100 colleges that offer some level of flight training in their curriculum. Many collegiate flight schools migrated to SMS in their organizations, but many schools have not yet transitioned to the new approach to safety management. It is important to understand how policies are adopted and implemented into the aviation industry, especially a policy as ubiquitous as SMS. This research begins to evaluate the status of SMS within collegiate flight training. 


\section{Research Questions}

The purpose of this study is to determine the current status of SMS development and implementation at collegiate flight schools across the US. The following three research questions are used to assess the status of the development and implementation of SMS at collegiate flight schools:

1. What are the SMS demographics of collegiate flight schools?

2. What level of organizational support is reported for SMS by collegiate flight schools?

3. What progress is being made toward the development or implementation of the components of SMS at collegiate flight schools?

\section{Background}

Aviation safety management has progressed through many changes in the last 50 years. Historically, safety management improved through a "fly-crash-fix-fly" approach (Stolzer et al., 2011), but the industry recognized that the public's perception of safety is based on the number of accidents that have occurred. Evolving approaches to safety management create an aviation system in which its users feel safe, and SMS is the most recent approach to safety management that attempts to be more proactive and predictive to improve aviation safety. ICAO requires member-states to develop and implement SMS within the various sectors of their aviation industry. The FAA is now following ICAO's lead by encouraging the aviation industry to adopt SMS.

\section{SMS Basics}

To understand the basic structure of SMS, a brief overview of the four components of an SMS and their elements need to be discussed. The four components of SMS are policy, safety risk management, safety assurance, and safety promotion (FAA, 2015). These components work together and contribute to developing a positive safety culture within the organization.

The first component of an SMS is policy. The management of an organization supports SMS by establishing policies and safety objectives for the organization. The policy developed by management should establish the direction and set the safety principles in action for the organization (Transportation Research Board, 2009). The second component of an SMS is safety risk management (SRM). A key philosophy within an SMS is to manage risk proactively. SRM seeks to identify hazards and systematically assess the risk associated with those hazards. Controls are then put into place to lower the risk to an acceptable level (Transportation Research Board, 2009). The third component of an SMS is safety assurance. Safety assurance ensures that "performance and effectiveness of safety risk controls and that the organization meets or exceeds its safety objectives through the collection, analysis, and assessment of information" (AC 120-92B, FAA, 2015b, p. 8). The final and fourth component of an SMS is safety 
promotion. The purpose of safety promotion is intended to support the efforts in developing a strong safety culture. Training, education, and other means of communication are key elements of safety promotion (Transportation Research Board, 2009).

\section{Origins of SMS}

SMS principles are used in different high-risk industries like the petroleum, nuclear, railway, marine, and chemical industries. These industries adopted SMS principles after experiencing a catastrophic tragedies, such as the Chernobyl accident, with the intention of addressing safety concerns before additional tragic events occurred (Transportation Research Board, 2007). The aviation industry is another high-consequence industry concerned with its safety record, especially as traffic volumes around the world continue to increase. SMS changes aviation safety-related policy making from a reactionary activity to a data-driven, proactive, and preventive policy-making activity.

\section{SMS throughout the Aviation Industry}

Different parts of the aviation industry are using SMS principles to manage their safety programs, although the level of involvement varies across the aviation industry. In all, FAA applies SMS principles to six different sectors of the aviation industry: (1) 121 operators; (2) Non-part 121 operators; (3) Maintenance, repair, and overhaul organizations (MROs); (4) Training organizations; (5) Design and manufacturing organizations; and (6) Airports. This section provides a brief discussion of the status of SMS within the US aviation system.

\section{SMS for 121 Operators}

The only sector with in the aviation industry to have SMS incorporated into regulation are those businesses certified under Federal Aviation Regulation (FAR) Part 121 - Operating Requirements: Domestic, Flag, and Supplemental Operations. Implemented in January, 2015, the new FAR Part 5 - Safety Management Systems, outlined the regulatory requirements for 121 operators to implement SMS into their operations.

\section{SMS for Airports}

Substantial effort has been dedicated to the development of SMS at the airports across the US. Although not yet regulatory, rulemaking activities have been underway since 2010 (Safety Management Systems for Certificated Airports, 2010). Investigation of the viability began even earlier in 2008 when the FAA sponsored many pilot programs at airports of varying size and complexity. The rulemaking for SMS for certificated airports has been through several different rulemaking extensions and revisions, and despite substantial effort over nearly a tenyear period to incorporate SMS into the airport environment, SMS for certificated airports remains unregulated.

Robertson, Harrison, and Ruiz (2014) conducted a similar study to determine the status of SMS implementation at FAR Part 139 Airports. The study indicated that the airport industry 
holds safety in high regard but is not convinced that SMS is a significant improvement to existing safety programs. This mindset has led to many airports not engaging in SMS until it becomes a regulatory requirement.

\section{Non-121 Operators, MROs, and Training Organizations}

The FAA provides a structure for voluntary implementation of SMS into other parts of the industry including non-121 operators, MROs, and training organizations. Using the Part 121 documentation as a framework, the FAA provides some SMS guidance in the form of gap analysis tools and Advisory Circular AC120-92B - Safety Management Systems for Aviation Service Providers, which is the same guiding documentation used by 121 certificated operators. In short, the FAA has not invested as much into developing SMS for these types of operators as they did when developing SMS for airlines and airports.

\section{Design and Manufacturing Organizations}

According to the FAA (2016), SMS principles are being incorporated into recent rulemaking efforts related to a Part 21 SMS. The FAA claims that a notice of proposed rulemaking for FAR Part 21 - Certification Procedures for Products and Articles, will be available some time during fiscal year 2018 (US Department of Transportation, 2016).

\section{Air Traffic}

The air traffic section of the aviation industry has been incorporating SMS principles and elements since 2007 (US Department of Transportation, 2007). Air traffic uses a fairly comprehensive set of guiding documents to help implement and develop their SMS programs. The requirements for SMS are detailed in the following series of orders: Order JO 1000.37, Air Traffic Organization Safety Management System Order JO 1030.1, Air Traffic Organization Safety Guidance (US Department of Transportation, 2014).

\section{Methodology}

This research used a survey instrument approved by an institutional review board to answer the three research questions. The population for this survey include safety officers at collegiate flight schools that are also members of the University Aviation Association (UAA), a non-profit organization involved in the advancement of degree-granting aviation programs from all segments of aviation. The UAA represents 97 post-secondary education institutions involved in aviation education. The convenient sample for the survey is comprised of the 55 active safety associated with the 97 UAA member institutions. The list of 55 safety officers consists of flight schools that operate some type of a safety program, whether it is a traditional safety program or an SMS. Two rounds of email to the population initiated participation in the study.

Advisory Circular (AC) 120-92B - Safety Management Systems for Aviation Operators provides a framework to aid in the development of the survey. Although 120-92B was designed 
to help airlines meet their regulatory requirements of SMS within Part 121 operations, the AC provides a succinct and comprehensive overview of SMS, its components, and the individual elements that drive SMS which form the basic structure of the survey. Robertson (2016) helped to fine tune the instrument utilizing the expertise of safety professionals within the flighttraining environment. In addition, colleagues that were considered safety specialists from Southern Illinois University, University of North Dakota, and Embry Riddle Aeronautical University reviewed the survey for validation.

\section{Calculating SMS Implementation}

Using AC 120-92B as a guide, this research developed a classification system to judge the degree to which a pilot training program has implemented SMS to manage their safety programs. Using the four components of SMS as a basic framework, the classification then adds the different elements that comprise each component of SMS. For example, a pilot training program that has implemented all four SMS components and all of their associated elements could be described as having a fully-implemented SMS. Further, this information can be used to calculate an overall degree of SMS implementation across the responding pilot training schools. For example, there are five different elements related to SRM. If all five elements of safety risk management are represented at all 28 institutions, the implementation score for SRM is calculated to be $100 \%$.

It is important to note the limitations of calculating the implementation scores. There are two factors that limit the application of the implementation score. First, the elements that are listed in Table 1 derive from AC 120-92B, which is a guiding rather than a regulatory document intended to help Part 121 operators and other aviation service providers to implement and manage an SMS. We cannot claim that this method of gauging SMS implementation is validated, but further investigation may reveal this method to be an adequate way to infer overall SMS implementation in pilot training schools or in any other sector of the aviation industry.

Similar to the first limitation, the second limitation is caused by the lack of uniformity of SMS elements across different organizations. For example, the survey asks respondents to identify all of the elements they use that relate to safety promotion. Among the items listed is the use of safety stand down as promotional element. While we can agree that a safety stand downs may be a useful promotional element, it is not required for a fully implemented SMS. This certainly limits the utility of an implementation score of $100 \%$, but the scoring system remains useful as a general guide to judge the overall implementation status of SMS and its components. Table 1 displays the four components of SMS and their associated elements that safety programs need to employ to implement SMS. It is important to note that there are elements and processes listed for safety promotion that are not necessary for SMS implementation. 
Table 1

SMS Components and Related Elements

$\underline{\text { Safety Policy }}$

Completed gap analysis

Implementation plan

Safety policy statement

SMS objectives

Identified accountable executive

Identified SMS manager/coordinator

Identified safety committee

Emergency planning and response

\section{$\underline{\text { Safety Risk Management }}$}

Hazard identification

Hazard tracking and documentation

Risk analysis

5-Step SRM process

Conducted safety risk assessments

\section{$\underline{\text { Safety Assurance }}$}

Confidential hazard reporting system - Web

Confidential hazard reporting system - Paper

Trend analysis

Safety performance monitoring

Continuous monitoring of safety controls

Flight data monitoring analysis

SMS audits or evaluations

Safety culture assessments

\section{$\underline{\text { Safety Promotion }}$}

Specialized SMS training

Regular SMS training - Employees

Regular SMS training - Students

Safety bulletin boards

Safety newsletters

Employee safety meetings

Student safety meetings

Safety awards program

Safety stand-downs

\section{Results}

The survey is divided into three different sections that, collectively, answer the three research questions. The first portion of the survey relate to SMS demographics, the second 
section of the survey relates to the level of management commitment to SMS activities, and the third section of the survey relates to the progress collegiate flight schools have made toward implementing SMS.

The two rounds of email yielded participation from more than half of the population. Of the 55 safety officers emailed, 28 safety officers responded to the survey, generating a response rate of $51 \%$.

\section{Part 1 - SMS Demographics}

One purpose of this research is to collect general SMS demographic information about collegiate flight programs. This type of information is useful as we monitor the propagation of SMS throughout the aviation industry. Just as Robertson et al., (2014) explored the spread of SMS throughout the airport sector, the current research aims to extend this line of research to monitor SMS in collegiate flight schools.

\section{Basic Institutional Demographics.}

The survey was used to discover the general demographic information related to SMS at collegiate flight schools. The respondents first provided information about basic school demographic information such as the name of the institution, whether their institution is certificated as a Part 141 pilot school, and whether their institution provides flight instruction or uses a third-party to provide their flight training. The names of the participating institutions were de-identified to keep the participants' responses confidential. Of the 28 survey respondents, 26 respondents indicated that their institutions classify as a Part 141 pilot school. Finally, the majority of the respondents $(89.14 \%)$ indicated that they provide their own flight training to pilots and do not contract their flight training out to another entity.

\section{General SMS Demographic Information.}

To gather SMS demographic information about the participating institutions, the next part of the survey asked participants to indicate their level of SMS knowledge. They also provided information that described the degree to which SMS is currently being used at the participating pilot training programs. Last in this portion of the survey, the respondents indicated when they expect their organizations to implement SMS.

Table 2 displays the level of familiarity the respondents have with SMS. Of the 28 safety officers-respondents, $24(85.71 \%)$ indicate that they are at least knowledgeable about SMS. 
Table 2

Safety Officer Knowledge of SMS

\begin{tabular}{lc}
\hline Degree of SMS Familiarity & $n$ \\
\hline No Knowledge & 0 \\
Some Knowledge & 4 \\
Knowledgeable & 11 \\
Very Knowledgeable & 13 \\
SMS Expert & 0
\end{tabular}

The participants describe their level of involvement to varying degrees. Table 3 displays the results the respondents provided for this survey question. At the time the surveys were submitted, only five respondents $(17.86 \%)$ believe they have a fully implemented SMS. The majority of the population have not yet fully implemented SMS in their pilot training programs. Only six $(21.43 \%)$ participants are not using SMS to manage their safety programs, which indicates that the majority of participants have adopted SMS to manage their safety programs.

Table 3

\section{SMS Involvement}

Degree of SMS Development/Implementation $\quad n$

SMS is Not Under Development 6

SMS is Under Development 9

Most SMS Components/Elements are in Place 8

SMS is Fully Implemented 5 
The last SMS demographic question asked the survey respondents to project when their organization plans to adopt a fully implemented SMS. Table 4 displays the projections of when the organizations plan to implement an SMS and when they expect their organizations to have an SMS fully in place. It is important to note that this question did not apply to $9(32.14 \%)$ of the respondents because they either have a fully implemented SMS or have no intention on further SMS development.

Table 4

Projected SMS Implementation

\begin{tabular}{lc}
\hline Number of Years Until Full SMS & $n$ \\
\hline Not Applicable & 9 \\
Within 1 year & 8 \\
Within 2 to 3 Years & 9 \\
More than 3 Years & 1 \\
Did Not Answer & 1 \\
\hline
\end{tabular}

\section{Part 2 - Management Commitment}

The second section of the survey aims to assess the commitment of the managers at the responding pilot training schools to SMS. In this case, management commitment is measured by assessing various management activities related to SMS and by asking survey participants to judge their managers' level of commitment to SMS.

\section{SMS-related Activities.}

The first question in this section asked respondents to identify all of the various SMS activities that indicate commitment to implementing and managing an SMS. Table 5 shows the distribution of the various SMS activities the respondents employ at their pilot training schools. 
Table 5

SMS Activities at Pilot Training Schools

\begin{tabular}{lc}
\hline SMS Activity & $n$ \\
\hline Invests human and financial resources & 19 \\
Proactive in preventing accidents & 25 \\
Consistently enforces safety procedures & 24 \\
Views regulatory violations seriously & 26 \\
Involved in safety activities & 24 \\
\hline
\end{tabular}

Because there were 28 respondents to the survey, each activity has a maximum participation level $(n)$ of 28 . None of the activities were represented by all 28 respondents, but all of the SMS activities are used by the majority of the research participants.

\section{Management's Commitment to Implement SMS.}

The safety officers responding to the survey were asked to evaluate their managers' level of commitment to implement SMS at their institution using a rating scale ranging from 0 to 10 , with 0 representing no commitment and 10 representing full commitment. There were 28 responses to this question with answers varying from $0-10$ with a Mean of 7.5 and a Median of $8.00(\mathrm{SD}-2.47)$.

\section{Part 3 - SMS Implementation}

The last portion of the survey aims to assess the level of implementation of SMS at the participating institutions. The four components of SMS and the associated elements provide the framework used to assess the level of SMS implementation. The level of implementation of SMS is measured by evaluating the different components and elements of SMS being used at the participating schools.

\section{Safety Policy.}

This section of the survey asked respondents to identify the elements related to safety policy that they use in their safety programs. Table 6 displays the results of this inquiry. Twentyfour of the 28 respondents $(85.71 \%$ ) had identified an SMS Manager or Coordinator and had emergency planning and response procedures in place. The least occurring element was the completion of a gap analysis with a total of eight replies $(28.57 \%)$. One result from this inquiry indicates that most of the safety policy related elements are represented by a majority of the 
responding organizations. A percentage of overall implementation of the safety policy component across the pilot training schools is calculated to be $64.73 \%$.

Table 6

Safety Policy Implementation

\begin{tabular}{ll}
\hline Safety Policy Activity/Process & $n$ \\
\hline Completed gap analysis & 8 \\
Developed an implementation plan & 14 \\
Developed a safety policy statement & 22 \\
Developed a set of SMS objectives & 18 \\
Identified an accountable executive & 19 \\
Identified an SMS manager/coordinator & 24 \\
Identified a safety committee & 16 \\
Developed an emergency response plan & 24 \\
\hline Total Safety Policy Implementation Score & $64.73 \%$
\end{tabular}

\section{Safety Risk Management.}

This section askes respondents to examine their SMS to identify the elements and processes that they had that relate SMS. Table 7 displays the results of this question. Of the 28 participants, $24(85.71 \%)$ have a method or methods in place for identifying hazards, and $50 \%$ of the respondents indicated that they have performed safety risk assessments. Only six respondents have a formalized 5-step SRM process established at their institution. The overall implementation score across all 28 pilot training programs is $57.86 \%$ implemented. 
Table 7

SRM Implementation

\begin{tabular}{ll}
\hline SRM Activity/Process & $n$ \\
\hline Hazard Identification & 24 \\
Hazard Tracking and Documentation & 18 \\
Risk Analysis & 19 \\
5-step SRM Process & 6 \\
Safety Risk Assessment & 14 \\
\hline Total SRM Implementation Score & $57.86 \%$
\end{tabular}

\section{Safety Assurance.}

The next question assessed the level of implementation of safety assurance activities at the responding institutions. To do this, the survey respondents identified which elements and processes that they had relating to the safety assurance component. Table 8 displays the results of this inquiry along with the implementation score for the safety assurance component. 
Table 8

Safety Assurance Implementation

\begin{tabular}{ll}
\hline Safety Assurance Activity/Process & $n$ \\
\hline Confidential Hazard Reporting System - Paper & 16 \\
Confidential Hazard Reporting System - Web & 15 \\
Trend Analysis Capability & 14 \\
Safety Performance Monitoring & 9 \\
Continuous monitoring of Safety Controls & 12 \\
Flight Data Monitoring Analysis & 8 \\
SMS Audits/Evaluations & 7 \\
Safety Culture Assessments & 18 \\
\hline Total Safety Assurance Implementation Score & $44.20 \%$
\end{tabular}

Twenty-six of the 28 respondents indicated that they had some type of confidential hazard reporting system. Four of the respondents indicated that they had both a paper based and web based system to report hazards. The elements of safety assurance are implemented at a rate of approximately $44 \%$ of the responding pilot training schools.

\section{Safety Promotion.}

The implementation of safety promotion activities is the last component assessed for this research. Table 9 displays the results of this survey question. Twenty-five (89.28\%) respondents reported that they have employee safety meetings but only eight $(28.57 \%)$ indicated that they have regularly scheduled training for their employees. Ten respondents indicated that they engage in safety stand downs. Typically, this involves closing down to discuss safety issues or have safety related training. Of these 10, only two indicated that they have regularly scheduled training and meetings for both students and employees. One particular school that does not organize regularly scheduled safety meetings or training did indicate that their safety stand down is one full safety day with a keynote speaker and 25-30 breakout sessions. Safety 
promotion activities are present $48.02 \%$ of the safety promotion activities are implemented across the sample of pilot training programs.

Table 9

Safety Promotion Implementation

Safety Promotion Activity/Process n n

\begin{tabular}{ll}
\hline Specialized SMS Training & 10
\end{tabular}

Regular SMS training - Employees 8

Regular SMS training - Students $\quad 11$

Safety bulletin boards $\quad 23$

$\begin{array}{ll}\text { Safety newsletters } & 11\end{array}$

Employee safety meetings $\quad 25$

$\begin{array}{ll}\text { Student safety meetings } & 19\end{array}$

Safety awards program $\quad 4$

$\begin{array}{ll}\text { Safety stand-downs } & 10\end{array}$

Total Safety Promotion Implementation Score $\quad 48.02 \%$

\section{Discussion}

\section{Research Question 1: What are the SMS demographics of collegiate flight schools?}

The first research question aims to find out about basic demographic information of the pilot training schools. It seems that knowledge of SMS is prevalent in the collegiate flight training industry as 24 of the 28 respondents indicated they were either knowledgeable or very knowledgeable about SMS. This knowledge appears to translate into implementation as 5 of the 28 respondents indicated they had a fully function SMS and 17 had one under development and most expect to be fully implemented within 3 years. These findings would seem to indicate that organizations choose to develop SMS once the processes and the intentions of SMS are understood. The collegiate flight training industry seems to be adopting SMS as their new safety standard as nearly all respondents either have or expect to have a fully implemented SMS within the next three years. 
Two areas of interest arise when these data are analyzed. The first reveals a reluctance to change institutional safety programs. Six respondents indicated they were not currently developing SMS and five had fully implemented SMS, but nine indicated that their timeline to implementation was "N/A." For the five institutions that have fully implemented SMS, this response makes sense, but it also indicates that four of the six that have not begun SMS development seem to have no intention to start. There could many reasons why organizations choose not to begin SMS implementation, but one could be lack of knowledge. Fully half of those respondents indicating SMS was not under development indicated only "some knowledge" of SMS and only one was "very knowledgeable." SMS seems to be gaining traction in the collegiate flight training industry, but there appears to be a knowledge gap between those choosing to implement and not, and some organizations that will not change unless mandated to do so.

The second interesting area involves a comparison between airports certified under FAR part 139 and these collegiate flight training providers. Neither group is mandated to comply with FAR Part 5, but there have been rumors for some time that both groups will eventually need to comply. The findings of Robertson et al., (2014) reveal a similar circumstance to that found in collegiate flight training. Those with knowledge of SMS seem to be willing to implement it even before the mandate is given, but there are also some hold out organization that will wait until they are mandated to comply before beginning the development and implementation process. This tendency seems to be less pronounced in the flight training industry when compared to the Part 139 airports. While there are certainly parallels, it seems that the collegiate flight training providers are more willing to adopt SMS than their airport counterparts.

\section{Research Question 2: What level of organizational support is reported for SMS by Collegiate Flight Schools?}

While the other two questions examined the implementation and acceptance of SMS, this question is different because it concerns the propensity for management to engage new ideas and change their organizations. Based on the survey, respondents indicated that the management of their organizations were primarily concerned with areas that could have detriment on the financial standing of the organization, such as regulatory violation $(n=26)$, accident prevention $(n=25)$, enforcing safety procedures and being involved in organizational safety activities $(n=24, n=24)$. In contrast, there were fewer that were willing to invest in human capital to improve their organization $(n=19)$. This indicates that the management of these organizations are interested in the safety of their operations from a fiduciary standpoint rather than investing in them to promote growth. This should not be misconstrued as a bad thing as safety in any flight training organization is paramount. Management at the respondent organizations do seem willing to pursue SMS implementation, and seem willing to provide

funding to do so. Nearly half of respondents reported using a software program of some sort to manage their safety program, and $69 \%$ of those use a commercial product or one developed 
in house. These two options require a capital investment by organizational management toward to management of a safety program and improving organizational safety. I would seem that flight training organizations are following similar paths to other industries by investing in technology and programmatic material over investing in their people and human capital.

\section{Research Question 3: What progress is being made toward the development or implementation of the components of SMS at Collegiate Flight Schools?}

The third question posed by this study involved the implementation of the components of SMS by collegiate flight schools. This is a broader question than simply SMS implementation as it concerns use of pieces of the SMS puzzle as well as the entire puzzle itself. To understand how SMS components are implemented by various collegiate flight schools, it is beneficial to examine each of the four major areas of SMS and see how components of those areas can be used.

\section{Safety Policy}

Safety policy in SMS determines the direction an organization will take with respect to safety programs. Safety policy activities are the first step in SMS implementation, and so it would be expected that most programs have elements of safety policy, which is what the data shows. Nearly all safety programs will have some form of policy statement, and we see that borne out in these data as 22 of the 28 respondents have safety policy statements and 24 have plans for emergency response. Most of the respondents have a safety coordinator of some sort $(\mathrm{n}=24)$, but far less have a full safety committee $(\mathrm{n}=16)$. Only eight respondents had completed a gap analysis, a precursor tool used to guide the implementation of SMS. While not required, use of a gap analysis can make the implementation of SMS easier for organizations. The safety policy areas that have been accepted into common use in the collegiate flight training industry are valuable, and it is the safety policy component that helps lay the foundation for SMS implementation.

\section{Safety Risk Management}

Safety risk management is the SMS component that deals with hazard identification and tracking as well as risk assessment and mitigation. Most respondents indicated they conducted hazard identification $(n=24)$, but only $3 / 4$ of those $(n=18)$ reported tracking and documenting those hazards to see hazard trends over time. It seems surprising that tracking hazard mitigation over time is not as common since it is important to track changes over time to understand if corrections were effective, but this could also be due to insufficient data to analyze. Trend analysis is one of the last stages in safety risk management because data must be collected over time. This could explain why fewer organizations have engaged in trend analysis.

\section{Safety Assurance}


Safety assurance deals with the ability to correct problems over time and continuously improve operational safety. This component also involves activities such as surveying organizational culture, instituting confidential hazard reporting, trend analysis, and tracking of performance indicators. These activities, and those like them within this component, are essential to the success of SMS over time, but safety assurance activities are typically conducted toward the end of SMS implementation. Much as we expect to see most organizations with safety policy initiatives, since it is the first step, we expect to see far less implementation of safety assurance, since it is the last step in SMS implementation. The most commonly implemented element was surveys of organizational culture $(n=18)$, followed by confidential hazard reporting either by electronic $(n=16)$ or paper $(n=15)$. These would seem to indicate that many collegiate flight training providers are actively seeking to encourage a robust safety culture and help to do so by establishing a means for non-punitive hazard reporting. This also seems to indicate that all or nearly all the respondents have some sort of confidential hazard reporting, whether that is electronic, paper, or both. Only half of the respondents conduct trend analysis of hazards over time to see if their corrections are effective, and even fewer actively monitored their safety controls $(n=12)$ or safety performance indicators $(n=9)$. While many of the elements of SMS have been implemented in collegiate flight training, safety assurance seems to be missing critical buy in from organizations, which can lead to problems because the entire purpose of safety assurance elements is to assure that the safety program is functioning the way it should. It is possible that these data are too preliminary and that, with time, safety assurance will be a more robust portion of the SMS process in these organizations.

\section{Safety Promotion}

The safety promotion component of SMS ensures that all principle parties are aware of the safety program and its goals. There were few respondents that indicated they conduct SMS specific training with employees or students, but that is to be expected when only five respondents have a fully implemented SMS. It is encouraging that almost 90 percent of respondents have employee safety meetings $(n=25)$ and safety bulletin boards $(n=23)$ to make information publicly available. Additionally, 19 respondents hold safety meetings specifically with their students so that they are aware of the safety program and why it is important. It is encouraging to see the level of safety promotion that has been adopted throughout the collegiate flight training industry, as presented by the survey respondents. The greatest safety program in existence does no good if no one knows about it. 


\section{Conclusions and Recommendations}

This research has shed light on the current state of SMS implementation within Collegiate flight training providers. While the sample is not large $(\mathrm{N}=28)$, the $51 \%$ response rate is within accepted margins for a survey of this nature (Baruch, 1999). SMS is being implemented in collegiate flight training much as it is by Part 139 compliant airports. SMS has not been mandated by either group, and so some are adopting early while others wait for a regulatory impetus to implement. About three-quarters of the collegiate flight training provider's responses indicated some level of SMS implementation, with nearly as many fully implemented as refusing to do so.

While this is interesting research, there are many areas of SMS at flight training providers which need to be explored, such as: What is the status of SMS implementation within the entire flight training industry? What role does general SMS knowledge play in organizational decision to implement SMS? How is SMS used to help understand and predict safety incidents or accidents? Is there a difference in how SMS is implemented at Part 61 flight training providers versus part 141 providers?

This study, if replicated, would benefit from some changes to the survey instrument to allow for more detailed information regarding the SMS elements and processes implemented at organizations. This instrument provided an overview, but lacked specifics as to how each item was being implemented. Additionally, more information collection on the quality and timing of safety promotion activities and the level of management commitment to SMS would further add to this important area of research.

SMS represents a shift in the understanding of safety. With SMS, safety awareness moves away from reacting to problems into a position of foreseeing and predicting where incidents will occur and actively trying to mitigate the hazards that lead to those incidents. It is heartening that the collegiate flight training industry has embraced SMS to this extent, but there is still more that can be done to improve aviation safety. 


\section{References}

Baruch, Y. (1999). Response rate in academic studies: A comparative analysis. Human Relations, 52(4), pp. 421-438. doi: 10.1177/001872679905200401

Federal Aviation Administration. (2015a). Safety Management Systems. Retrieved from https://www.faa.gov/about/initiatives/sms/explained/basis/\#evolution

Federal Aviation Administration. Department of Transportation, Flight Standards Service. (2015b). Advisory Circular AC 120-92B, Safety management systems for aviation service providers. Washington, DC: Author.

International Civil Aviation Organization (ICAO). (2013) Safety management manual (SMM) (Document 9859). Montreal, Quebec, Canada: International Civil Aviation Organization.

Pilot Career Center. (2016). College and university programs in USA. Retrieved from http:/ / www.pilotcareercenter.com/Pilot-Training-Flight-SchoolCategory/USA/2/FlightCollege

Robertson, M. F. (2016). Safety professional's perception of the relationship between safety management systems and safety culture. Journal of Aviation Technology and Engineering, 6(1). http://dx.doi.org/10.7771/2159-6670.1137

Robertson, M. F., Harrison, B., \& Ruiz, J. (2014). The Status of Safety Management Systems at FAR Part 139 Airports. Collegiate Aviation Review, 32(2), 51.

Safety Management Systems for Certificated Airports, 75 Fed. Reg. 62008 (October 7, 2010) (to be codified at 14 C.F.R. pt. 139.

Stolzer, A. J., Goglia, J., \& Halford, C. D. (2011). Implementing safety management systems in aviation. Surrey, England: Ashgate.

Transportation Research Board. Airport Cooperative Research Program (ACRP). (2009). Safety management systems for airports. Volume 2, Guidebook. Washington, DC: Author.

Transportation Research Board, Airport Cooperative Research Program (ACRP). (2007). Safety management systems for airports. Volume 1, Overview. Washington, DC: Author.

US Department of Transportation, Federal Aviation Administration, Air Traffic Organization. (2007). Air Traffic Organization policy: Air Traffic Organization en route and oceanic services safety management systems (FAA Order JO 1000.39). Retrieved from https://www.faa.gov/documentLibrary/media/Order/ND/JO\%201000.39.pdf

US Department of Transportation, Federal Aviation Administration, Air Traffic Organization. (2014). Safety management system manual version 4.0: Air Traffic Organization 2014. Retreived from https://www.faa.gov/air_traffic/publications/media/faa_ato_SMS_ manual_v4_20140901.pdf 
US Department of Transportation, Federal Aviation Administration. (2016, July 15). Safety management system - SMS for design and manufacturing organizations. Retrieved from https://www.faa.gov/about/initiatives/sms/specifics_by_aviation_industry_type/desi gn_and_manufacturing_organizations/ 